\section{Avaliação do valor de corte de TSH em amostras de filtro na triagem neonatal para diagnóstico de hipotireoidismo congênito no Programa "Primeiros Passos" - IEDE/RJ}

\author{
Evaluation of TSH cutoff value in blood-spot samples in neonatal \\ screening for the diagnosis of congenital hypothyroidism \\ in the Programa "Primeiros Passos" - IEDE/RJ
}

Bianca Barone', Clarisse Lourandes da Silva Lopes', Latife Salomão Tyszler', Valéria Borges do Amaral', Rosa Helena C. Zarur', Valéria Neto Paiva', Denise Boechat Leite', Ricardo M. R. Meirelles'

\section{RESUMO}

Objetivo: Analisar casos de hipotireoidismo congênito $(\mathrm{HC})$ confirmados ou não, triados pelo Programa "Primeiros Passos", estratificando-os em faixas de TSH em filtro (TSH-F). Materiais e métodos: Estratificar, em faixas deTSH-F em função doTSH em soro (TSH-S), os casos convocados para teste confirmatório de janeiro/2006 a julho/2009. Resultados: Cerca de $37 \%$ dos casos confirmados (475) apresentaram TSH-F $\geq 9,5 \mathrm{mUi} / \mathrm{L}$, mas a maioria dos casos confirmados estava nas faixas de TSH-F mais baixas. Entre os casos não confirmados (4.613), a maior parte se encontrava nas faixas mais baixas. Não houve faixa deTSH-F exclusiva dos casos não confirmados. Conclusão: $\mathrm{O}$ valor de corte do TSH-F utilizado é fundamental no diagnóstico do $\mathrm{HC}$ e deve ser baixo, mesmo que sejam realizados mais testes confirmatórios. Mais estudos são necessários para determinar o melhor valor de corte de TSH-F para triagem neonatal. Arq Bras Endocrinol Metab. 2013;57(1):57-61

\section{Descritores}

Hipotireoidismo congênito; triagem neonatal; TSH; doenças congênitas

\begin{abstract}
Objective: To analyze the confirmed or not-confirmed cases of neonatal screening $(\mathrm{CH})$ screened in the Programa "Primeiros Passos", stratifying them intoTSH blood-spot (TSH-BS) ranges. Materials and methods: To stratify, in ranges of TSH-BS as a function of TSH serum (TSH-S), the cases called for a confirmatory test from January, 2006 to July, 2009. Results: Around 37\% of the confirmed cases (475) showed TSH-F $\geq 9.5 \mathrm{mUi} / \mathrm{L}$, but most of the confirmed cases were in lower TSH-F ranges. Among the unconfirmed cases $(4,613)$, most were found in the lower ranges. There was no TSH-F range exclusive to unconfirmed cases. Conclusion: TSH-BS cutoff value used is crucial in the diagnosis of $\mathrm{CH}$ and should be low, even if more confirmatory tests are performed. More studies are needed to determine the best cutoff value of TSH-BS for neonatal screening. Arq Bras Endocrinol Metab. 2013;57(1):57-61
\end{abstract}

\section{Keywords}

Congenital hypothyroidism; neonatal screening; TSH; congenital diseases
'Programa "Primeiros Passos", Instituto Estadual de Diabetes e Endocrinologia Luiz Capriglione (IEDE), Rio de Janeiro, RJ, Brasil

Correspondência para: Bianca Barone Rua Moncorvo Filho, 90 20211-340 - Rio de Janeiro, RJ, Brasil bbarone@terra.com.br

Recebido em 1/Abr/2012 Aceito em 21/Ago/2012 


\section{INTRODUÇÃO}

$\mathrm{O}$ hipotireoidismo congênito (HC) é uma patologia que decorre da redução (ou ausência) da ação dos hormônios tireoideanos no organismo (1), podendo promover o surgimento de retardo metal, atraso neuropsicomotor (2) e déficit estatural $(3,4)$. Seu diagnóstico por meio do rastreamento neonatal e início do tratamento precoce previne suas consequências $(5,6)$. Atualmente, recomenda-se que o tempo adequado entre o nascimento e o início do tratamento seja de até 14 dias, principalmente nos casos mais graves de $\mathrm{HC}$ (3,4,7-11). Contudo, no caso do Brasil, sabe-se que esse tempo adequado nem sempre é atingido, em função de diversos fatores (12-16).

A incidência do HC varia ao redor do mundo, sendo no Brasil relatada a ocorrência em 1:3.694 nascidos vivos (17). As manifestações clínicas do HC no período neonatal podem passar despercebidas, o que faz com que o rastreamento neonatal seja uma ferramenta tão importante para o diagnóstico precoce dessa enfermidade (18).

A dificuldade diagnóstica precoce com base exclusiva em parâmetros clínicos e o potencial de prevenção do retardo mental secundário ao HC são as bases da criação da triagem neonatal para essa patologia. Em 1974, em Quebec e Pittsburg, foi primeiramente instituído o rastreamento neonatal para $\mathrm{HC}(19,20)$.

Apenas em 2001, o Ministério da Saúde brasileiro criou o Programa Nacional de Triagem Neonatal (PNTN) (portaria $n^{\circ} .822$ de $06 / 06 / 2001$, artigo $1^{\circ}$, parágrafo $\left.1^{\circ}\right)$, estabelecendo fases sucessivas de inclusão de patologias para triagem. A Fase I inclui apenas fenilcetonúria (PKU) e HC. A Fase II inclui PKU, HC e hemoglobinopatias (HGP). Já a Fase III é composta de PKU, HC, HGP e fibrose cística (2l). Atualmente, o Rio de Janeiro (RJ) encontra-se em Fase III do PNTN (22). O Programa "Primeiros Passos" é uma das unidades de triagem neonatal cadastradas no RJ, sendo responsável, atualmente, por 75 municípios.

Apesar de o HC estar incluído em programas de triagem neonatal ao redor do mundo, não há um consenso universal sobre um valor de corte de TSH em filtro (TSH-F) ideal a ser utilizado. Isso faz com que haja grande variação dos valores de corte de TSH-F em função do país estudado e até mesmo dentro de um mesmo país. Apesar da falta de consenso, com o passar dos anos, os valores de corte de TSH-F utilizados pelos diversos programas de triagem neonatal passaram a ser cada vez mais baixos $(1,7,12,13,19,23-25)$.
O objetivo deste trabalho é levantar, retrospectivamente, os casos de HC triados em nosso Serviço, no período de janeiro/2006 a julho/2009, após mudança de corte no valor de TSH-F, realizada para aumentar a sensibilidade do método, avaliando o impacto que isso ocasionaria na triagem neonatal para o diagnóstico de HC.

\section{MATERIAIS E MÉTODOS}

Trata-se de um estudo retrospectivo, com levantamento dos dados dos testes de triagem neonatal realizados pelo Programa "Primeiros Passos" no período de janeiro/2006 a julho/2009. O trabalho em questão foi aprovado pelo Comitê de Ética e Pesquisa do IEDE, sob o número 2808/Parecer 02908.

Entre o segundo e o quinto dias de vida dos recémnascidos foram coletadas as amostras por punção do calcanhar direto no verso do papel-filtro, com aproximadamente $12 \mathrm{~mm}$ de diâmetro, com encaminhamento para subsequente análise $(26,27)$.

As dosagens de TSH-F foram realizadas com kit AutoDelfia ${ }^{\circledR}$ (PerkinElmer, Finlândia, ensaio imunofluorimétrico tempo-resolvido) (28), enquanto para os testes de TSH confirmatório em soro (TSH-S) e T4 livre (em sangue periférico) utilizaram-se kits Beckman Coulter ${ }^{\circledR}$ (quimioluminescência, Access) (29).

Em nosso programa de triagem neonatal, a partir de junho de 2006, passamos a utilizar um valor de corte de TSH-F $\geq 4,5 \mathrm{mUi} / \mathrm{L}$ (sangue total), com o objetivo de aumentar a sensibilidade do método. Anteriormente, utilizávamos um valor de corte de $10 \mathrm{mUi} / \mathrm{L}$ (sangue total). As crianças que apresentaram resultados acima desse valor de 4,5 mUi/L foram reconvocadas para realização de teste confirmatório em soro. A confirmação do HC foi feita quando TSH-S (em soro) encontravase $\geq 10 \mathrm{mUi} / \mathrm{L}$. Nesses casos, foi iniciado tratamento apropriado com levotiroxina, na dose de 10-15 $\mu \mathrm{g} /$ $\mathrm{kg} /$ dia, mantendo-se essa criança em acompanhamento periódico no Serviço, com realização de dosagens de T4 livre e de TSH para eventual reajuste da dose dessa medicação.

Os dados levantados foram: data de nascimento e data de coleta da amostra de sangue em filtro (Teste do "pezinho"), com cálculo do intervalo entre nascimento e coleta neonatal; resultado de TSH-F e do TSH-S (apresentados como média, mínimo e máximo). Foram compilados os testes de TSH-F realizados nos períodos estudados e o número de casos reconvocados para 
realização de teste confirmatório em soro. Além disso, estratificamos as crianças em diversas faixas de resultado de TSH em filtro em função do resultado do teste confirmatório em soro.

Os dados compilados foram estruturados em uma base de dados, utilizando-se o programa Excel (versão 2007) (Microsoft ${ }^{\circledR}$ ) e estão apresentados como média \pm desvio-padrão ou mediana. As análises foram feitas com o uso do Programa de Estatística SPSS (Statistical Package for Social Sciences) para Windows, versão 11.

\section{RESULTADOS}

Foram realizados 523.344 testes de TSH em filtro no período, dos quais 5.639 encontravam-se alterados, sendo esses casos então reconvocados para realização de teste de confirmação em soro. Destes, 551 não compareceram para realizar o teste confirmatório, apesar de os responsáveis terem sido acionados pelas vias legais. Desse modo, analisamos aqui os resultados dos 5.088 casos que compareceram para realizar o teste confirmatório. A mediana do tempo entre nascimento e coleta da amostra em filtro dos casos estudados foi de 5 dias (variando de 2 a 515 dias). Desses testes alterados em filtro, em 90,7\% dos casos convocados não se confirmou a alteração quando realizada dosagem em soro.

A média do TSH-F nos casos de HC confirmados foi de 29,22 $\mathrm{mUi} / \mathrm{L}$ (variando de 4,50 a 569,82 $\mathrm{mUi} / \mathrm{L})$. Já no caso dos $\mathrm{HC}$ não confirmados, a média de TSH-F foi de $6,30 \mathrm{mUi} / \mathrm{L}$ (variando de 4,50 a $221,62 \mathrm{mUi} / \mathrm{L})$. Em relação ao TSH confirmatório (em soro), a média nos casos de HC confirmados foi de 99,20 mUi/L (variando de 10,00 a 2179,54 mUi/L), enquanto nos casos de $\mathrm{HC}$ não confirmados essa média foi de $4,61 \mathrm{mUi} / \mathrm{L}$ (variando de 0,10 a 9,99 ). Em relação ao T4 livre do exame confirmatório, a média nos casos confirmados foi de $1,03 \mathrm{ng} / \mathrm{dL}$ (variando de 0,01 a $2,05 \mathrm{ng} / \mathrm{dL}$ ) e, nos casos de HC não confirmados, foi de $1,24 \mathrm{ng} / \mathrm{dL}$ (variando de 0,39 a $2,50 \mathrm{ng} / \mathrm{dL}$ ).

Apesar de a faixa com o maior número de casos confirmados ser a de TSH-F $\geq 9,5 \mathrm{mUi} / \mathrm{L}(37,4 \%)$, uma expressiva parcela de grupo com TSH-S $\geq 10,0 \mathrm{mUi} / \mathrm{L}$ apresenta TSH-F nas faixas mais baixas $(24,4 \% \mathrm{com}$ TSH-F entre 4,5 e $5,4 \mathrm{mUi} / \mathrm{L} ; 15,6 \%$ entre 5,5 e 6,4 $\mathrm{mUi} / \mathrm{L} ; 10,6 \%$ entre 6,5 e $7,4 \mathrm{mUi} / \mathrm{L} ; 7,2 \%$ entre $7,5 \mathrm{e}$ $8,4 \mathrm{mUi} / \mathrm{L}$ e 4,8\% entre 8,5 e 9,4 mUi/L). Já nos casos com TSH-S $<10,0 \mathrm{mUi} / \mathrm{L}$, a maior parte dos pacientes se encontra nas faixas mais baixas de TSH-F ( $49,3 \%$ entre 4,5 e $5,4 \mathrm{mUi} / \mathrm{L})$. Nas faixas subsequentes, houve um decréscimo progressivo do número de indivíduos $(24,6 \%$ com TSH-F entre 5,5 e $6,4 \mathrm{mUi} / \mathrm{L} ; 12,2 \%$ entre 6,5 e $7,4 \mathrm{mUi} / \mathrm{L} ; 5,8 \%$ entre 7,5 e $8,4 \mathrm{mUi} / \mathrm{L} \mathrm{e}$ $3,5 \%$ entre 8,5 e $9,4 \mathrm{mUi} / \mathrm{L})$. Cerca de $4,5 \%$ dos casos com TSH-F entre 4,5 e 4,9 mUi/L apresentaram TSH-S $\geq 9,5 \mathrm{mUi} / \mathrm{L}$. As crianças com TSH-F alterado que apresentaram TSH-S elevado ( $\geq 10,0 \mathrm{mUi} / \mathrm{L})$ estão sendo avaliadas prospectivamente quanto à possibilidade de se tratar de hipertireotropinemia neonatal. A figura 1 apresenta a distribuição dos casos com TSH-S (ditos confirmados) em função dos resultados de TSH-F. Não há uma faixa de TSH em filtro na qual apenas se encontrem casos de HC não confirmados.

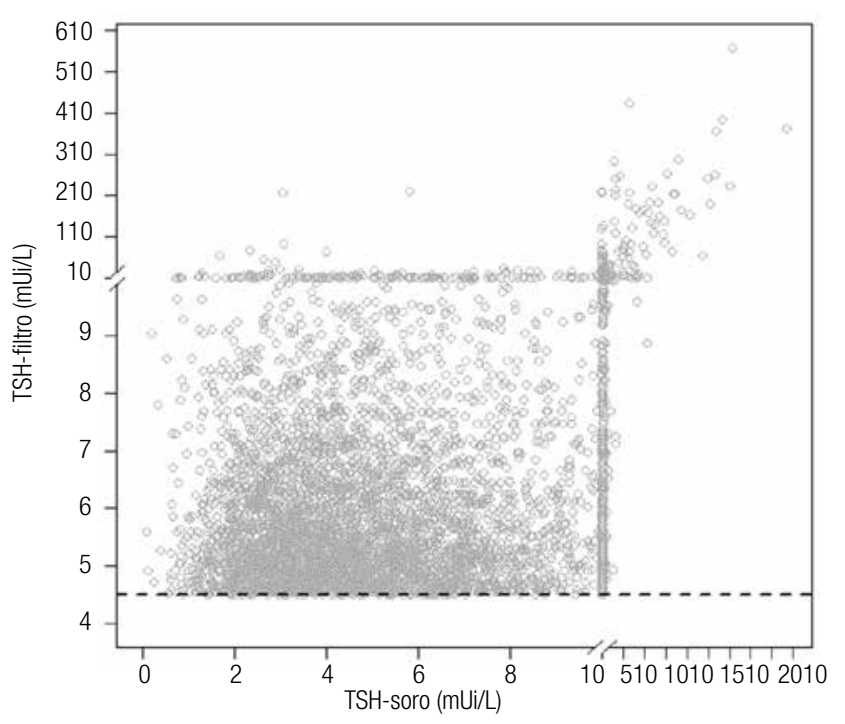

TSH: hormônio estimulador da tireoide.

Figura 1. Distribuição dos testes de TSH em soro em função do resultado do teste confirmatório em filtro.

\section{DISCUSSÃO}

Um ponto que deve ser considerado nos programas de triagem neonatal para $\mathrm{HC}$ é o uso de valores de corte mais baixos de TSH-F, de modo a aumentar a sensibilidade do teste, mesmo que à custa da realização de quantidade adicional de testes confirmatórios. Isso porque a escolha de um valor de TSH-F mais baixo permite que mais crianças sejam encaminhadas para a realização de um teste confirmatório e que sejam captadas crianças com valores anteriormente considerados normais. Apesar de vários programas de triagem neonatal estarem reduzindo seus valores de corte de TSH-F ao longo dos anos (1,7,12,13,19,23-25), não há uniformidade ou consenso de um valor de TSH-F a ser utilizado no Brasil ou no mundo. A maioria dos serviços que faz uso de ensaios imunofluorimétricos utili- 
za valores de corte de TSH-F próximos a 10,0 mUi/L $(1,16,17,19,30,31)$, enquanto uma publicação do Serviço de Triagem Neonatal de Sergipe utilizou um valor de corte de 5,2 mUi/L (25). Ainda assim, o valor de corte utilizado por nós foi menor que os demais existentes na literatura nacional e internacional.

Já foi demonstrado que ocorre redução fisiológica dos níveis séricos de TSH com o aumento da idade dos recém-nascidos até uma estabilização dos valores (32). Isso pode influenciar a escolha de um único valor de corte de TSH, já que ainda há no Brasil uma grande variação nas idades de coleta das amostras de TSH-F e de TSH-S para os testes confirmatórios $(12,14,15,30,33)$. Contudo, sugere-se padronizar a época de coleta das amostras, em vez de utilizar valores de corte variados $(30,33)$. Além da coleta muito precoce da amostra de TSH-F, outros podem ser os fatores responsáveis por resultados falso-positivos desse exame, como o caso de prematuridade, gemelaridade ou presença de retardo de crescimento intrauterino $(34,35)$. Em nosso trabalho, não temos o levantamento de todos esses dados. Apenas dispomos dos dados de idade na ocasião da coleta da amostra para TSH-F, o que limita que possamos excluir que o elevado número de casos de TSH-F alterado se deva a algum desses fatores perinatais.

Estudos mais recentes têm mostrado que crianças com alteração no TSH-F e que no TSH confirmatório apresentam resultado discretamente alterado (entre 6,0 e 9,9 mUi/L) podem vir a ter algum grau de comprometimento neurológico, sendo cogitado o acompanhamento laboratorial dessas crianças e, eventualmente, aventada a necessidade de instituição de terapia com levotiroxina (19). Contudo, esse assunto é ainda muito controverso e mais estudos são necessários para que esses resultados dos casos de hipertireotropinemia neonatal transitória alterem as rotinas de triagem neonatal.

O valor de corte do TSH-F utilizado para diagnóstico é de suma importância no diagnóstico dessa enfermidade e deve ser baixo o suficiente para reduzir o número de casos falso-negativos, mesmo que para tanto sejam realizados mais testes confirmatórios. Sugerimos que o valor de corte de TSH-F para a triagem neonatal de $\mathrm{HC}$ seja de 4,5 mUi/L, já que em nosso levantamento foi possível encontrar uma expressiva quantidade de casos que apresentaram essa faixa de TSH-F que foram posteriormente confirmados com TSH-S $\geq 10$ $\mathrm{mUi} / \mathrm{L}$. Mais estudos são necessários para determinar se esse é realmente o melhor valor de corte de TSH para que as metas da triagem neonatal sejam atingidas.

Agradecimentos: à Secretaria Estadual de Saúde do Rio de Janeiro e aos funcionários do Programa "Primeiros Passos", que se dedicam diariamente e de modo incansável na busca por auxiliar as crianças por nós triadas.

Declaração: os autores declaram não haver conflitos de interesse científico neste estudo.

\section{REFERÊNCIAS}

1. Silva LO, Dias VM, Silva IN, Chagas AJ. Hipotireoidismo congênito transitório: perfil das crianças identificadas no Programa Estadual de Triagem Neonatal de Minas Gerais, Brasil. Arq Bras Endocrinol Metabol. 2005;49(4):521-8.

2. Bottler J. Repercussões neurológicas do hipotireoidismo congênito. Arq Bras Endocrinol Metabol. 1996;40(4):264-70.

3. Bongers-Schokking JJ, Koot HM, Wiersma D, Verkerk PH, de Muinck Keizer-Schrama SM. Influence of timing and dose of thyroid hormone replacement on development in infants with congenital hypothyroidism. J Pediatr. 2000;136(3):292-7.

4. Fisher DA. The importance of early management in optimizing IQ in infants with congenital hypothyroidism. J Pediatr. 2000; 36(3):273-4.

5. Allen DB, Hendricks SA, Sieger J, Hassemer DJ, Katcher ML, Maby $\mathrm{SL}$, et al. Screening programs for congenital hypothyroidism. How can they be improved? Am J Dis Child. 1988;142(2):232-6.

6. LaFranchi S. Congenital hypothyroidism: etiologies, diagnosis, and management. Thyroid. 1999;9(7):735-40.

7. Pharoah PO, Madden MP. Audit of screening for congenital hypothyroidism. Arch Dis Child. 1992;67(9):1073-6.

8. Rovet JF. Long-term neuropsychological sequelae of early-treated congenital hypothyroidism: effects in adolescence. Acta Paediatr Suppl. 1999;88(432):88-95.

9. Dubuis JM, Glorieux J, Richer F, Deal CL, Dussault JH, Van VG. Outcome of severe congenital hypothyroidism: closing the developmental gap with early high dose levothyroxine treatment. J Clin Endocrinol Metab. 1996;81(1):222-7.

10. Revised guidelines for neonatal screening programmes for primary congenital hypothyroidism. Working Group on Neonatal Screening of the European Society for Paediatric Endocrinology. Horm Res. 1999;52(1):49-52.

11. Toublanc JE. Guidelines for neonatal screening programs for congenital hypothyroidism. Working Group for Neonatal Screening in Paediatric Endocrinology of the European Society for Paediatric Endocrinology. Acta Paediatr Suppl. 1999;88(432):13-4.

12. Nascimento ML, Pires MMS, Nassar SM, Ruhland L. Avaliação do Programa de Rastreamento Neonatal para Hipotireoidismo Congênito da Secretaria de Estado da Saúde de Santa Catarina. Arq Bras Endocrinol Metabol. 2003;47(1):75-81.

13. Ramalho RJ, Valido DP, Aguiar-Oliveira MH. Avaliação do Programa de Triagem para o Hipotireoidismo Congênito no Estado de Sergipe. Arq Bras Endocrinol Metabol. 2000;44(2):157-61.

14. Ramos AJS, Rocha AM, Costa ADM, Benecio AVL, Ramos ALC, Silva CRA, et al. Avaliação do Programa de Rastreamento de Doenças Congênitas em Campina Grande - PB, Brasil. Arq Bras Endocrinol Metabol. 2003;47(3):280-4.

15. Stranieri I, Takano OA. Avaliação do Serviço de Referência em Triagem Neonatal para Hipotireoidismo Congênito e Fenilceto- 
núria no Estado de Mato Grosso, Brasil. Arq Bras Endocrinol Metabol. 2009;53(4):446-52.

16. Ramalho RJ, Ramalho AR, Oliveira $C R$, Aguiar-Oliveira MH. Evolução do Programa de Triagem Neonatal para Hipotireoidismo Congênito e Fenilcetonúria no Estado de Sergipe de 1995 a 2003. Arq Bras Endocrinol Metabol. 2004;48(6):890-6.

17. de Carvalho TM, dos Santos HP, dos Santos IC, Vargas PR, Pedrosa J. Newborn screening: a national public health programme in Brazil. J Inherit Metab Dis. 2007;30(4):615.

18. Setian N. Hipotireoidismo congênito. In: Setian N, editor. Endocrinologia Pediátrica. Aspectos Físicos e Metabólicos do Recém-nascido ao Adolescente. São Paulo: Sarvier; 2002. p. 259-68.

19. Rose SR, Brown RS. Update of newborn screening and therapy for congenital hypothyroidism. Pediatrics. 2006;117(6):2290-303.

20. LaFranchi S. Detecção neonatal de hipotireoidismo congênito: uma história de sucesso? Arq Bras Endocrinol Metabol. 1995;39:80-8.

21. Ministério da Saúde do Brasil. Portaria Ministerial número 822 de 6 de Junho de 2001.

22. Ministério da Saúde do Brasil. Programa Nacional deTriagem Neonatal - Habilitação do Rio de Janeiro - Portaria SAS № 491, de 23 de outubro de 2001.

23. Vasilevskaia IA, Guzeev GG, Baikov AD, Rykina IA, Bugrova VB. [Clinical aspects of screening and diagnosis of congenital hypothyroidism in neonates in Moscow]. Probl Endokrinol (Mosk). 1993;39(4):25-7.

24. American Academy of Pediatrics AAP Section on Endocrinology and Committee on Genetics, and American Thyroid Association Committee on Public Health: newborn screening for congenital hypothyroidism: recommended guidelines. Pediatrics. 1993;91(6):1203-9.

25. Ramalho AR, Ramalho RJ, Oliveira CR, Santos EG, Oliveira MC, Aguiar-Oliveira MH. Programa de Triagem Neonatal para Hipotireoidismo Congênito no Nordeste do Brasil: Critérios Diagnósticos e Resultados. Arq Bras Endocrinol Metabol. 2008;52(4):617-27.

26. Ministério da Saúde do Brasil. Manual de Normas Técnicas e Rotinas Operacionais do Programa Nacional de Triagem Neonatal. 2004.

27. Programa "Primeiros Passos" de Triagem Neonatal I-HGdEdRdJSdEdS. Orientações gerais sobre Coleta e Busca Ativa. 2006.

28. PerkinElmer Life and Analytical Sciences WO. AutoDELFIA Neonatal hTSH -Time-resolved fluoroimmunoassay. Instructions for use. [B032-312]. 2006. Turku, Finlândia.

29. Bayer S.A. Produtos Diagnósticos. Manual de Ensaios ACS 180. 2000.

30. Gruters A, Krude H. Update on the management of congenital hypothyroidism. Horm Res. 2007;68 Suppl 5:107-11.

31. Nesi-França S, Pereira RM, Lara F, Pelaez JM, Morizaki TMY, Ditzel EC, et al. Triagem Neonatal para Hipotireoidismo Congênito no Estado do Paraná - Avaliação de 601 casos detectados em 14 anos. Rev Med Minas Gerais. 2005;15(2):83.

32. Fisher DA, Klein AH. Thyroid development and disorders of thyroid function in the newborn. N Engl J Med. 1981;304(12):702-12.

33. Nesi-França S, Domingos MT. Triagem neonatal do hipotireoidismo congênito: novas conquistas... novos desafios... Arq Bras Endocrinol Metabol. 2008;52(4):579-80.

34. Korada M, Pearce MS, Avis E, Turner S, Cheetham T. TSH levels in relation to gestation, birth weight and sex. Horm Res. 2009;72(2):120-3.

35. Ng SM, Wong SC, Paize F, Chakkarapani E, Newland P, Isherwood D, Didi M. Multivariate analyses of factors that affect neonatal screening thyroid stimulating hormone. J Pediatr Endocrinol Metab. 2011;24(9-10):727-32. 